\section{Alfabetización en salud y adherencia farmacológica en adultos mayores con enfermedades crónicas, de la ciudad de Temuco}

\author{
CARLA SOLEDAD FIGUEROA SAAVEDRA ${ }^{1, \mathrm{a}, \mathrm{c}}$, \\ TAMARA FRANCISCA OTZEN HERNÁNDEZ2,b,d, \\ VIVIANA FIGUEROA DELGADO ${ }^{1, a}$, PATRICIO SANHUEZA ${ }^{1, a}$, \\ JEREMY CANDIA ${ }^{1, a}$
}

\section{Association between health literacy and adherence to medications among older adults}

Background: Lack of compliance with medication prescription is common among older adults and hamper an adequate management of chronic conditions. Aim: To study the association between health literacy and medication adherence in older adults with chronic diseases in Temuco, Chile. Material and Methods: The Health Literacy survey for Spanish-speaking Adults, MiniMental examination and the Morisky-Green test to assess compliance with medication prescription were applied to 119 older adults aged 60 to 88 years. Results: All participants had an adequate global cognitive performance; $24 \%$ had inadequate literacy, and $42 \%$ did not comply with medication prescription. There was a significant correlation between health literacy and medication adherence. Conclusions: Health literacy influences medication adherence in Chilean older adults.

(Rev Med Chile 2020; 148: 653-656)

Key-words: Aged; Chile; Chronic Disease; Health Literacy; Medication Adherence.

\author{
${ }^{1}$ Facultad de Ciencias de la Salud, \\ Universidad Autónoma de Chile. \\ Temuco, Chile. \\ ${ }^{2}$ Doctorado en Ciencias \\ Médicas, Facultad de Medicina, \\ Universidad de La Frontera. \\ Temuco, Chile. \\ ${ }^{a}$ Fonoaudiólogo. \\ bPsicólogo. \\ ${ }^{\mathrm{C} M s c .}$ \\ ${ }^{\mathrm{d}} \mathrm{PhD}$ \\ Una parte de la presente \\ investigación fue financiada a \\ través de los fondos de apoyo \\ a la investigación en temáticas \\ de inclusión, iniciativa de la \\ oficina de gestión de la inclusión \\ y diversidad cultural de la \\ Universidad Autónoma de Chile, \\ Sede Temuco. \\ Los autores declaran no tener \\ conflictos de interés. \\ Recibido el 11 de abril de 2019, \\ aceptado el 26 de mayo de 2020 . \\ Correspondencia a: \\ Tamara Francisca Otzen \\ Hernández \\ Universidad de La Frontera, \\ Temuco, Chile. \\ Avenida Francisco Salazar 1145, \\ Temuco \\ tamara.otzen@ufrontera.cl
}

$\mathrm{E}$ n Chile la población de adultos mayores va en aumento, se estima que al 2020 los adultos mayores de 65 años alcanzarán $17,7 \%$ de la población total, lo que se traduce en un reto para el sistema de salud ${ }^{1}$. Este segmento es donde se encuentran la mayor cantidad de enfermos crónicos ${ }^{2}$, siendo estas enfermedades las responsables de $68 \%$ de la mortalidad general en nuestro país ${ }^{3}$, cifra mayor que la referida a nivel mundial ${ }^{2}$.

Dentro de la atención de la multimorbilidad surge un concepto asociado muy relevante que se denomina Alfabetización en Salud (AS). Definido como "las habilidades sociales y cognitivas que determinan el nivel de motivación y la capacidad de una persona para acceder, entender y utilizar la información de forma que le permita promover y mantener una buena salud" 4 . Existe evidencia que propone que la población que posee menor nivel de AS constituye un segmento vulnerable, por tener deficiencias en el reconocimiento de los síntomas de enfermedad, la aplicación de medidas de prevención, la utilización adecuada de medicamentos y la posibilidad de establecer una satisfactoria relación médico paciente ${ }^{5}$. De esta forma el bajo nivel de AS representa una epidemia silenciosa ${ }^{6}$ que podría afectar especialmente a la población en este rango de edad, considerando los cambios a nivel cognitivo al que está expuesto este grupo ${ }^{7}$.

Considerando lo anterior es que se estableció el objetivo de estudiar la asociación entre AS y 
adherencia farmacológica en adultos mayores con enfermedades crónicas de la ciudad de Temuco.

\section{Material y Método}

El diseño fue de corte transversal, la muestra contempló un total de 119 individuos pertenecientes a la ciudad de Temuco, elegidas a través de un muestreo por conveniencia. Dentro de los instrumentos de evaluación, se aplicaron: 1) Short Assessment of Health Literacy for Spanish Adults $\left(\right.$ SAHLSA-50) ${ }^{8}$; 2) Test Minimental ${ }^{9}$; y 3) Test de Morisky-Green ${ }^{10,11}$.

En cuanto a los procedimientos de la investigación, en primer lugar, se elaboró el protocolo de investigación, el cual fue aprobado por el Comité de Ética de la Carrera de Fonoaudiología, de la Universidad Autónoma de Chile, asignándole el código FONOAUD0019. Posterior a la lectura y firma de un consentimiento informado, se aplicaron los instrumentos a los adultos mayores.

Los datos fueron ingresados al programa Statistical Package for Social Science (IBM SPSS) versión 23. Posteriormente, se utilizó estadística exploratoria a través de pruebas de normalidad de Kolmogorov-Smirnov con las variables continuas, AS y Adherencia Farmacológica, arrojando como resultado un valor de $\mathrm{p}<0,01$, por lo que se procedió a realizar un análisis descriptivo utilizando medianas y rangos, el análisis inferencial se realizó por medio de la prueba de correlaciones de Spearman y correlación parcial controlada por polifarmacia (4 o más) y deterioro cognitivo (menos de 24 puntos en Minimental), considerando un nivel de confianza del 95\%.

\section{Resultados}

La muestra constituida por 119 adultos mayores presentó un promedio de edad de 71 años [60-88]. Todos los sujetos presentaron a lo menos una enfermedad crónica y en el consumo de medicamentos el $21 \%$ de los sujetos consumen diariamente 4 o más. La enfermedad crónica que apareció con mayor frecuencia fue la hipertensión $(72,3 \%)$. En relación con las características generales de desempeño cognitivo en los adultos mayores, se observó un desempeño bajo en 9,2\% de ellos, $23,5 \%$ mostró una baja AS con una mediana de 43 [24-50]. Un 41,2\% de los sujetos no se adhieren a la terapia farmacológica obteniendo una mediana general de $0[0-4]$, en este punto es importante mencionar que para efecto de interpretación de valores se invirtió la escala de puntuaciones donde a mayor puntaje se observó una mayor adherencia (Tabla 1).

Según los resultados obtenidos se puede afirmar que existe relación significativa entre la AS y la adherencia farmacológica, siendo esta relación media y positiva, es decir, en la medida que aumenta el conocimiento acerca de los conceptos asociados a salud aumenta la adherencia

Tabla 1. Descripción de desempeño en alfabetización en salud, adherencia farmacológica y desempeño cognitivo global en adultos mayores

\begin{tabular}{|c|c|c|c|}
\hline Porcentajes & & & Mediana (rango) \\
\hline Edad & & & $71[60-88]$ \\
\hline Alfabetización en Salud & & & $43[24-50]$ \\
\hline Alfabetización en Salud & $\begin{array}{l}\text { Inadecuada } \\
\text { Adecuada }\end{array}$ & $\begin{array}{l}23,5 \% \\
76,5 \%\end{array}$ & $\begin{array}{l}- \\
-\end{array}$ \\
\hline Cantidad de enfermedades & & & $2[1-4]$ \\
\hline Cantidad de medicamentos & $\begin{array}{l}\geq 4 \\
<4\end{array}$ & $\begin{array}{l}21 \% \\
79 \%\end{array}$ & \\
\hline Adherencia farmacológica & $\begin{array}{l}\text { Se adhiere } \\
\text { No se adhiere }\end{array}$ & $\begin{array}{l}58,8 \% \\
41,2 \%\end{array}$ & - \\
\hline Desempeño cognitivo global & $\begin{array}{l}\text { Con DC } \\
\text { Sin DC }\end{array}$ & $\begin{array}{r}9,2 \% \\
90,8 \%\end{array}$ & $\begin{array}{l}- \\
-\end{array}$ \\
\hline
\end{tabular}


Cognición y fármacos en adultos mayores enfermos crónicos - C. Figueroa et al

Tabla 2. Correlación entre alfabetización en salud, adherencia farmacológica, desempeño cognitivo global cantidad de enfermedades y consumo de medicamentos

\begin{tabular}{|lccc|}
\hline & & Alfabetización en Salud & Adherencia farmacológica \\
Alfabetización en Salud & $r_{s}$ & 1,00 & $0,42^{* *}$ \\
Desempeño cognitivo global & $r_{s}$ & $0,44^{* *}$ & 0,17 \\
Cantidad de enfermedades & $r_{s}$ & 0,03 & 0,13 \\
Cantidad de medicamentos & $r_{s}$ & 0,18 & $0,23^{*}$ \\
\hline
\end{tabular}

${ }^{* *} p<0,001 .{ }^{*} p<0,005$.

farmacológica $\left(r_{s}=0,34 ; p<0,01\right)$. Por otra parte, al eliminar la influencia del consumo de 4 o más medicamentos por día, sigue existiendo una asociación significativa entre ambas $\left(r_{p}=0,29\right.$; $\mathrm{p}<0,01)$. Así también, al controlar la asociación según deterioro cognitivo también existe asociación entre la AS y la adherencia farmacológica $\left(\mathrm{r}_{\mathrm{p}}=0,33 ; \mathrm{p}<0,01\right)$ (Tabla 2).

\section{Discusión}

La presente investigación tuvo entre sus principales hallazgos la asociación encontrada entre la AS y adherencia farmacológica, esto quiere decir que a medida que disminuye la AS es menor el nivel de adherencia farmacológica observada en los adultos mayores con enfermedades crónicas de la ciudad de Temuco, al respecto Turner et al. ${ }^{12}$, reportó que los adultos mayores con hipertensión y menor conocimiento sobre su enfermedad, tenían menos probabilidades de adherirse a su terapia antihipertensiva. De igual modo Lee et al. ${ }^{13}$, indagó acerca de la relación entre la AS y la adherencia farmacológica en los adultos mayores con enfermedades crónicas, concluyendo que la AS era un predictor de la adherencia farmacológica. Lo que finalmente concuerda con los resultados de esta investigación. Sin embargo, también hay evidencia que da cuenta de que no existe asociación entre la AS y la adherencia farmacológica ${ }^{14}$, en donde se consideraron también pacientes mayores de 60 años, todos con hipertensión, pero sin considerar el número de medicamentos, lo que podría explicar estas diferencias, siendo de todas formas cuidadosos en las interpretaciones que se realicen, considerando siempre la población en estudio.

En relación con la adherencia farmacológica en este estudio se obtuvo un porcentaje de $58,8 \%$, esta cifra es un poco más alta a la de otro estudio reportado en nuestro país, sin embargo, estos estudios estaban enfocados solamente a un tipo de enfermedad crónica ${ }^{15,16}$.

En este estudio se encontró $23,5 \%$ de nivel inadecuado de AS, lo que es ligeramente más bajo a lo reportado por Konfino ${ }^{6}$ acercándose más a las cifras de países desarrollados ${ }^{17}$.

En cuanto a la adherencia farmacológica, en esta investigación se encontró que al agrupar a los sujetos entre aquellos que consumen más de tres medicamentos de aquellos que consumen menos, la relación entre AS y la adherencia a farmacológica no cambia, lo que debe ser tomado con cuidado considerando el sesgo de esta investigación asociado a la forma del reporte del número de medicamentos consumidos. Independiente de esto, es importante considerar el número de medicamentos al evaluar la asociación entre AS y la adherencia a farmacológica, ya que los adultos mayores frecuentemente se ven expuestos a la utilización de polifarmacia ${ }^{17}$, lo que genera un mayor entrenamiento previo, ya que en su mayoría se manifiestan una multiplicidad de síntomas y/o trastornos ${ }^{18}$.

Por consiguiente, los resultados reflejan la necesidad de abrir nuevas líneas de investigación que consideren la información aquí obtenida y la incorporación del uso de las tecnologías en el abordaje de la adherencia terapéutica.

La AS podría ser un elemento gravitante en la adherencia farmacológica de los adultos mayores con enfermedades crónicas, sin embargo, no es el único elemento que debe ser estudiado, también es importante considerar otros elementos como las características sociodemográficas, psicológicas, cantidad de medicamentos y enfermedades crónicas que posee un adulto mayor. Pudiendo de esta forma generar un modelo más complejo que 
tribute al éxito de un tratamiento en la población de adultos mayores.

Cabe recalcar que existen varios instrumentos para evaluar alfabetización en salud, por lo que la elección del test SAHLSA-50 por sobre otros, se debió a que este instrumento es el único que a la fecha cuenta con adecuadas propiedades psicométricas (validez de constructo y confiabilidad) para adultos chilenos ${ }^{8}$, sin desmerecer que pudieran evaluarse este constructo con otros instrumentos.

Es importante mencionar que el tamaño de la muestra es limitado y no se realizaron estimaciones para determinar el número de sujetos, declarándose como potencial sesgo de investigación por la falta de representatividad de sus resultados y la imposibilidad de extrapolación de estos.

Por otra parte, esta investigación tiene limitaciones en cuanto al registro de los datos, en donde lamentablemente no se registró el número de medicamentos consumidos, la frecuencia del consumo de medicamentos, el tiempo de duración de la enfermedad, entre otros, siendo por consiguiente imposible considerar factores sumamente importantes como polifarmacia, siendo este uno de los principales factores asociado a la adherencia farmacológica ${ }^{19}$, lo que se espera mejorar en futuros estudios.Este estudio representa un acercamiento entre la AS y la adherencia farmacológica, evidenciando la necesidad de generar programas que fortalezcan las estrategias de autocontrol, monitoreo y efectividad terapéutica.

\section{Referencias}

1 Comisión Económica para América Latina y el Caribe (CEPAL), Observatorio Demográfico. Santiago: 2016.

2 WHO. 10 datos sobre las enfermedades no transmisibles. Report of a WHO Consultation enfermedades no transmisible. Geneva, Marzo 2013.

3 Solimano G, Mazzei M. ¿De qué mueren los chilenos hoy?: perspectivas para el largo plazo. Rev Med Chile 2007; 135 (7): 932-8.

4 Simonds SK. Health education as social policy. Health Education Monograph. 1974; 2: 1-25.

5 Williams MV, Baker DW, Parker RM, Nurss JR. Relationship of functional health literacy to patients knowledge of their chronic disease Arch Intern Med 1998; 158 (2): 166-72.

6 Konfino J, Mejía R, Majdalani MP, Pérez-Stable EJ. Alfabetización en salud en pacientes que asisten a un hospital universitario. Medicina 2009; 69 (6): 631-4.

7 Rabadán, OJ. Lenguaje y envejecimiento: bases para la intervención. Masson, SA, 1998.

8 Monsalves MJ. Mañalich J, Fuentes E. Validación del test Short Assessment of Health Literacy for Spanish-speaking Adults en Chile, para medir alfabetización en salud. Rev Med Chile 2016; 144 (5): 604-10.

9 Quiroga LP, Albala BC, Klaasen PG. Validación de un test de tamizaje para el diagnóstico de demencia asociada a edad, en Chile. Rev Med Chile 2004; 132 (4): 467-78.

10 Morisky DE, Green LW, Levine DM. Concurrent and predictive validity of a self-reported measure of medication adherence. Med Care 1986; 24 (1): 67-74.

11 Val-Jiménez A, Amorós G, Martínez P, Fernández ML, León M. Estudio descriptivo del cumplimiento del tratamiento farmacológico antihipertensivo y validación del test Morisky y Green. Aten Primaria 1992; 10 (5): 767-70.

12 Turner BJ, Hollenbeak C, Weiner MG, Ten Have T, Roberts C. Barriers to adherence and hypertension control in a racially diverse representative sample of elderly primary care patients. Pharmacepidemiol Drug Saf. 2009; 18 (8): 672-81.

13 Yun-Mi Lee RN, Hye Yon Yu, Mi-Ae You, Youn-Jung Son. Impact of health literacy on medication adherence in older people with chronic diseases. Collegian. 2017; 24 (1): 11-8.

14 Rosas-Chávez G, Romero-Visurraga CA, Ramírez-Guardia E, Málaga G. El grado de alfabetización en salud y adherencia al tratamiento en pacientes con hipertensión arterial en un hospital nacional de Lima, Perú. Rev Peru Med Exp Salud Pública 2019; 36 (2): 214-21.

15 Bello N, Montoya P. Adherencia al tratamiento farmacológico en adultos mayores diabéticos tipo 2 y sus factores asociados. Gerokomos. 2017; 28 (2): 73-7.

16 Varleta P, Akel C, Acevedo M, Salinas C, Pino J, Opazo $\mathrm{V}$, et al. Prevalencia y determinantes de adherencia a terapia antihipertensiva en pacientes de la Región Metropolitana. Rev Med Chile 2015; 143 (5): 569-76.

17 Núñez AJ, Montiel A, Martín E, Torres B, Lara C, González JA. Adherencia al tratamiento en pacientes polimedicados mayores de 65 años con prescripción por principio activo. Atención Primaria 2005; 46 (5): 238-45.

18 Arriagada L, Jirón M, Ruiz I. Uso de medicamentos en el adulto mayor. Rev Hosp Clin Univ Chile 2008; (19): 309-17.

19 Koren G, Nordon G, Radinsky K, Shalev V. Clinical pharmacology of old age. Expert Rev Clin Pharmacol 2019; 12 (8): 749-55. 